\title{
TIL KORPUSLARI SEMANTIK TEGLARINI YARATISHDA SEMANTIK IZOH BERISH MUAMMOLARIGA DOIR
}

\section{Soliyev Muslimbek}

Alisher Navoiy nomidagi Toshkent davlat o'zbek tili va adabiyoti universiteti, Kompyuter lingvistikasi mutaxassisligi 1-bosqich magistranti nurikfariz@gmail.com

Annotatsiya: Ushbu maqolada til korpuslarining semantik teglar tizimini ishlab chiqish, semantik teglarni yaratishda semantik izohlar berish muammolari, semantik teglashning $\mathrm{o}^{\prime}$ zbek tili korpuslari uchun ahamiyati va afzalliklari xususida fikr yuritilgan.

Kalit so'zlar: semantik teg, so ‘z, matn, semantika, teglar tizimi.

KIRISH. Zamonaviy tilshunoslikni kompyuter texnologiyasisiz tasavvur qilish qiyin. Ayni holatni kompyuter lingvistikasida ham kuzatishimiz mumkin. Lingvistika yo "nalishida har tomonlama qulay, zamonaviy elektron lug'atlarning yaratilishi kompyuter oldida turgan muhim vazifalardan sanaladi. Chunki shu kungacha ma'lum bir doirada semantik teglash amalga oshirilgan bo'lsada Muammoning ilk bora tadqiqot obyekti sifatida tanlanishi mavzuning dolzarbligini ko'rsatadi.

$\mathrm{O}^{`}$ zbek tili korpuslari uchun semantik teglar tizimini ishlab chiqish uchun

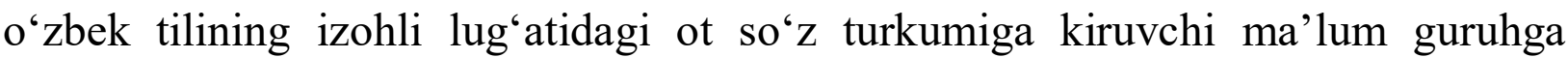
kiruvchi otlar uchun atributlar tizimini ishlab chiqish, ushbu teg guruhlarining ichiga shu ma'no-mazmunga ega bo'Igan so'zlarni joylashtirib chiqish va natijada dasturiy ta'minot yaratish dolzarb masala hisobladi.

$\mathrm{O}^{`}$ zbek tili korpuslari uchun semantik teglar tizimini ishlab chiqish hozirgi kunda o'zbek tilidagi so'zlarni ma'lum gurhlarga biriktiradi. Buning natijasida, ma'lum soha odamlari uchun bir guruhga kiruvchi so'zlarni oson topish va qo'llashda qulaylik yaratishdan iborat. Semantik teglashni quyida batafsil tushuntirib o'tamiz.

1. Qaldirg'och 1 - Chumchuqsimonlar turkumining bir oilasini tashkil etadigan, qanotli ingichka va uzun, juda tez uchadigan, qora-ola kichkina qushcha. Tuya qaldirg'och. Shahar qaldirg'ochi. Tog' qaldirg'ochi. Bir necha qaldirg'och Salorning oqishi bo ylab uchar va uchgan qo yi "valfajr" o qir edilar. A.Qodiriy. 
"O'tgan kunlar"

-in - Qush, hasharot va hayvonlarning yashash, bola ochish uchun qurgan joyi, uya:

- Oyijon, qarang, uyimizga qaldirg'ochlar in qurishyapti. Demak, biz bilan birga yasharkan-da, - dedi ko zlarini katta-katta ochib.

(https://gulxan.uz/hikoyalar/qaldirgochlar-qaytadi)

uchmoq - Qanot vositasida havoda harakatlanmoq, qaqnot qoqib, biror tomonga yo'nalmoq; parvoz qilmoq.

Ilon bir chang solib, uning tilini uzib olibdi. Bir payt qaldirg'och uchib kelib qolibdi. Ilon: Qani, sen ayt-chi, dunyodagi eng shirin go'sht nimaniki ekan? debdi. (http://erkatoy.uz/uzb/kutubxona/hikoyalar/2558-qaldirg-och-himmati) kelmoq - Yurish, uchish harakati yoki transport vositalari bilan so'zlovchiga qarama-qarshi tomon (joy)dan so'zlovchi turgan tomonga, joyga yo'l olmoq, yo"nalmoq; shu tomondagi biror joyga yetmoq Qaldirg'ochlar kela solib shoshilgancha o'z xonalariga kirib ketishdi, keyin tezda qaytib chiqib nimalardir deb chug'urlasha boshladi.

(https://n.ziyouz.com/portal-haqida/xarita/uzbek-nasri/maqsud-qoriyev-19262010/maqsud-qoriyev-qaldirg-ochlar-bahorda-keladi-hikoya) 2. Za'faron I - (arabcha - ziravor o'simlik, zafar) Ingichka uzun bargli, to' $q$ sariq gulli, piyozsimon ildizli o'simlik va uning gulidan tayyorlanadigan, ovqatni xushta'm va rangdor qiladigan ziravor. Za'faron o'rta asrlardan beri bahosi va qadri tushmay kelayotgan yagona ziravordir.

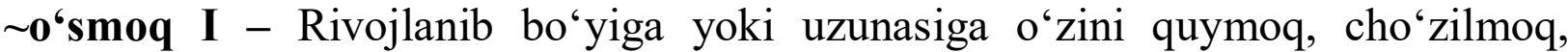
rivojlanmoq

Bola yaxshi o'sdi. Katta maydondagi g'o 'zalar barq urib o'syapti. Bodom daraxti yer tanlamaydi, toshloq, qumloq joylarda ham o saveradi.

〜'simlik - Havo va tuproqdagi organic va anorganik moddalar bilan oziqlanadigan, anorganik moddalarni organik moddalarga aylantirish xususiyatiga ega bo'lgan, odatda, biror joyga o'rnashgan holda rivojlanadigan organizm.

Za'faron - savsarguldoshlar oilasiga mansub subtropik o'simlik onaligining quritilgan tivitlaridir. Xalq tabobatida za'faronli choy yuragi zaif va nafasi qisadigan kasallarga ichirilgan. (https://avitsenna.uz/zafaron/)

〜dorivor - (forzcha - shifobaxsh, dori bo'ladigan) Turli kasalliklarga dori 
bo`ladigan yoki dori olinadigan

Zafaron (shafran) dorivor giyohining inson salomatligi uchun o 'ta muhim, foydali va shifobaxsh xususiyatlaridan xabardormisiz? (https://agrobaza.uz/uz/zafarondorivor-simligi/)

1. Do'ppi - Avra-astarli, ko'pincha qavima, pilta urilgan, tepa, kizak va jiyakdan tarkib topadigan guldor yoki gulsiz, to'garak yoki to'rtburchakshakldagi bosh kiyimi

Bosh omon bo 'lsa, do 'ppi topilar. (O`zbek xalq maqoli)

-bosh I - Tananing bo'yindan yuqorigi, oldingi (odamda, hayvonlarda) qismi; kalla

Do'ppi - yengil bosh kiyimi. Qadimdan eron va turkiy xalqlar orasida keng tarqalgan. Turkiston xalqlari orasida (ayniqsa O'zbekiston va Tojikiston hududida) milliy kiyim turiga aylangan. Boshqa xalqlar do'ppilaridalaridan o'zbek do'ppilarilari o'ziga xos shakli, bezagi bilan farqlanadi. (https://meros.uz/object/doppi)

-tikmoq I - Igna-ip bilan choklab ulamoq, biriktirmoq;

Shuningdek, do 'ppilar bayrambop, diniy va kundalik bo ladi. Ushbu milliy bosh kiyimi yumshoq yoki qattiq matodan tikilib, kashta yoki munchoqlar bilan bezatiladi, unga yumaloq yoki kvadrat shakl beriladi. (https://uzbekistan.travel/uz/o/ozbek-doppisi-sirlari/)

〜omon - (arabcha - havfsizlik; hotirjamlik; rahm-shafqat) Sog ${ }^{6}$-salomat, eson-sog ${ }^{6}$

Sharof Boshbekovning «Masxaraboz» filmida masxaraboz Tesha kaltak eb, keyin ust-boshini qoqa turib aytgan gapi bor: "Qo'l butun, oyoq ishlayapti, bosh ham soppa-sog'. Ie, do 'ppi qani? Ha, mana ekan, topdim. Bosh omon bo 'lsa, do 'ppi topiladi, degani shu bo 'lsa kerak...» Muallif xalqning dono naqlini o'z o'rnida qo 'llab, so 'z o 'yini qilgan. (http://mahalladosh.uz/oz/post?id=6822)

Semantik guruhlardagi so'zlarning izohlarini berish o'zbek tili korpuslari uchun va uning foydalanuvchilari uchun keng imkoniyat va qulayliklarni yuzaga chiqaradi. Semantik teg guruhlaridagi so'zlarga bog'lanadigan so'zlar va ularning qanday holatda bog 'lanib kelganligi misollar orqali ko'rsatib berilganligi bilan 
ham foydali.

\section{Foydalanilgan adabiyotlar:}

1. Ўзбек тилининг изохли луғати. Икки томлик. М., 1981.

2. Хожиев А. Ўзбек тили синонимларининг изохли луғати. - Тошкент. Ўқитувчи, 1974.

Хожиев А. Ўзбек тили синонимларининг изохли луғати. - Тошкент. Ўқитувчи, 1974.

3. Ўзбек тилининг изохли луғати (А.Мадвалиев тахрири остида). 5 жилдлик. - Тошкент: “Ўзбекистон миллий энциклопедияси” Давлат илмий нашриёти, 2000-2006.

4. Ahmedova D. "Atov birliklarining o'zbek tili korpuslari uchun leksik semantik teglashning lingvistik asos va modellari”; - T. 2008

5. Зализняк А.А. Русско-французский учебный словарь. - М., 1972.

6. https://n.ziyouz.com/kutubxona/category/10-o-zbek-tilining-izohli-lug-ati

7. http://idum.uz/uz/ 